\title{
Laringectomía parcial en cáncer laríngeo precoz: 25 años de experiencia
}

\section{Partial laryngectomy for early laryngeal cancer: A 25-year experience}

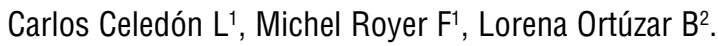

\begin{abstract}
RESUMEN
Introducción: La laringectomía parcial y la radioterapia son alternativas eficaces en el tratamiento de pacientes con cáncer laríngeo precoz, comparables tanto en tasas de sobrevida como en conservación laríngea.

Objetivo: Se describe la experiencia del primer autor en los últimos 25 años.

Material y método: Estudio retrospectivo de pacientes con cáncer laríngeo precoz tratados entre los años 1980 y 2005. Se analiza los antecedentes de los pacientes, tipo de tratamiento, histología del tumor, complicaciones y tasas de sobrevida.

Resultados: Se incluye un total de 96 pacientes, con una edad promedio de 58 años. 1 tipo histológico más frecuente fue el carcinoma epidermoide (92\%). 日 53\% se sometió a cirugía laríngea parcial y 47\% a radioterapia La intervención más habitual fue la laringectomía frontolateral (68\%). Las complicaciones más frecuentes fueron la presencia de granulomas (16\%) y falta de cierre del traqueostoma (16\%). Se constató recidiva en 4 pacientes tratados con radioterapia y 1 sometido a cirugía La sobrevida actual es de $91 \%$ para quienes recibieron radioterapia y $100 \%$ en quienes se practicó laringectomía parcial. Se presenta los resultados del tratamiento con o sin compromiso de la comisura anterior.

Discusión y conclusiones: La experiencia con ambas modalidades son comparables a la literatura, con complicaciones y secuelas esperables. La aparición de nuevas técnicas debe compararse en su realidad local con las terapias establecidas y la decisión terapéutica sigue correspondiendo a un acuerdo informado entreel tratante y su paciente.

Palabras clave: Cáncer laríngeo precoz, laringectomía parcial, radioterapia
\end{abstract}

\section{ABSTRACT}

Introduction. Partial laryngectomy and radiotherapy are good alternatives for early laryngeal cancer, as they are similar in both survival rate and laryngeal preservation.

Aim. The first author's 25-year experience on this subject is described.

Material and method. Retrospective review of early laryngeal cancer patients treated between 1980 and 2005. History, type of treatment, tumor histology, complications and survival rates are analyzed.

1 Médico Otorrinolaringólogo, Hospital Clínico de la Universidad de Chile.

2 Médico Cirujano, Universidad de Chile. 
Results. A total of 96 patients is included, with an average age of 58 years. The most frequent histological type was epidermoid carcinoma (92\%). 53\% of patients had partial laryngectomy, and $47 \%$ had radiotherapy. Most patients had a fronto-lateral laryngectomy (68\%), and the most frequent complications were granulomas (16\%) and failure of tracheostoma closure (16\%). Four radiotherapy patients and one that underwent surgery had recurrences. Present survival rate is $91 \%$ for radiotherapy and $100 \%$ for partial laryngectomy. Treatment results with and without anterior comissure involvement are presented.

Discussion and conclusions. Our experience with both treatment alternatives is comparable to that reported in the literature, with similar complications. New techniques must be compared with those currently used, taking into account local reality, and the therapeutic decision is still an informed agreement between the treating physician and the patient.

Key words. Early laryngeal cancer, partial laryngectomy, radiotherapy.

\section{INTRODUCCIÓN}

El cáncer de laringe es una de las neoplasias de cabeza y cuello más frecuentes, excluyendo a los carcinomas derivados de la piel ${ }^{1,2}$. Se presenta con mayor frecuencia en el género masculino, en la quinta y sexta décadas de la vida. Se ha considerado como factores de riesgo principales al tabaco ${ }^{2-4}$ y consumo de alcohol,5; también se ha descrito la infección por virus papiloma humano $0^{7,8} \mathrm{y}$ la presencia de reflujo faringo-laríngeo $0^{9,10}$, entre otros. El tipo histológico predominante es el epidermoide y, al momento del diagnóstico, el 50\% de los pacientes presenta una enfermedad localizada².

La denominación "cáncer laríngeo precoz" abarca los estadios I y II de la clasificación del American Joint Committee on Cancer Staging (AJCC), en los cuales el tumor no provoca fijación cordal, no compromete cartílago ni presenta enfermedad ganglionar. En el enfrentamiento terapéutico de estos tumores se privilegia una estrategia de tratamiento unimodal, cuyas alternativas pueden ser radioterapia (RT), laringectomía parcial (LP) 0 cirugía transoral con láser ${ }^{11,12}$. Debe recordarse que en tumores supraglóticos el esquema de tratamiento debe incluir el manejo linfático del cuello debido a la mayor probabilidad de metástasis regionales ocultas ${ }^{13}$.

La elección de la modalidad de tratamiento para el cáncer de laringe precoz puede ser difícil, dado que las terapias tienen eficacias semejantes. En este sentido, las tasas de sobrevida y conservación laríngea reportadas para RT y LP son similares en la mayoría de la literatura extranjera ${ }^{14-16}$. La aparición ulterior del láser como herramienta terapéutica también ha demostrado resultados comparables ${ }^{17-20}$. Por lo tanto, la decisión es producto, la mayoría de las veces, de un acuerdo entre el paciente y su médico tratante. Para esto se debe considerar las condiciones generales del sujeto, la extensión del tumor, su calidad vocal, su actividad, etc., ya que el resultado de esta evaluación influirá en la elección de la terapia. En general la RT tiende a ser el tratamiento de elección en el norte de Europa, Australia y Canadá; en cambio, se prefiere la cirugía al sur de Europa y muchos centros de USA ${ }^{21}$.

El compromiso de la comisura anterior (CA) en el cáncer de cuerda vocal es un problema aún no resuelto. Existe controversia acerca de cuál es la mejor terapia: RT o cirugía, sea ésta abierta 0 endoscópica con láser. En estos casos el uso de la RT, según Chen y Zouhair, no es buena ${ }^{22,23}$. Chen informa que la sobrevida a los 5 años en el cáncer glótico, con y sin compromiso de la CA, es de $55 \%$ y $90 \%$, respectivamente. En cambio, Stoeckli ${ }^{24}$, en cánceres de glotis tratados con RT no encuentra que la sobrevida dependa del compromiso de la CA. Con el uso del láser el problema es similar. Autores como Pradhan ${ }^{25}$ informan de una recurrencia del $28 \%$ si existe compromiso de la CA y sólo de $13 \%$ en caso contrario. Zeitel no encuentra diferencia en sus resultados, independiente de si está 0 no involucrada la $\mathrm{CA}^{26}$. Según Steiner, al igual que Zeitel, el control local en paciente con cáncer de cuerda vocal tratados con cirugía con láser no depende del compromiso de la $\mathrm{CA}^{27}$.

El objetivo de este trabajo es describir la experiencia del primer autor en el tratamiento de pa- 
cientes con cáncer laríngeo en etapa precoz de cuerda vocal, manejados con cirugía abierta 0 con RT, en los últimos 25 años. Importancia especial le asignamos al compromiso de la CA en la sobrevida $y / 0$ recidiva de estos pacientes.

\section{MATERIAL Y MÉTODO}

Se realizó un estudio retrospectivo en el que se incluyeron pacientes con cáncer laríngeo, en etapas in situ, I y II según la AJCC, tratados entre los años 1980 y 2005 por el primer autor. Durante ese período se aplicó siempre el mismo protocolo: a) en los carcinomas in situ se realizó RT; b) en los T1 de cuerda se efectuó RT; cuando éstos comprometían la CA se practicó cirugía parcial frontolateral con resección de la comisura; c) en los T2 de cuerda siempre se hizo cirugía frontolateral.

En esta oportunidad se revisaron sólo los carcinomas de cuerda vocal, ya que nos interesaba saber la importancia que tiene en el resultado final el compromiso de la CA, y por otro lado homogeneizar la población a estudiar. Se excluyen ex profeso los carcinomas de la supraglotis, ya que su conducta biológica, diagnóstico, pronóstico y tratamiento, difieren de los de ubicación glótica.

Se revisaron las fichas clínicas de los pacientes confeccionando un formulario individual con datos demográficos, antecedentes generales, consumo de tabaco y alcohol, diagnóstico previo de laringitis crónica, antecedentes de RT o cáncer previo. Se consignó la ubicación y el estadio tumoral, diagnóstico histológico, modalidad de tratamiento, técnica quirúrgica, complicaciones precoces y tardías, recidiva, conservación laríngea y sobrevida a cinco años.

\section{RESULTADOS}

Se incluyeron 96 pacientes con diagnóstico de cáncer laríngeo precoz, $80 \%$ de sexo masculino, con una relación hombre:mujer de 4:1, siendo la edad promedio de 58 años. Dentro de los factores de riesgo descritos para cáncer laríngeo presentes en esta serie, el hábito tabáquico fue el más frecuente, encontrado en el $94 \%$ de los pacientes. El consumo de alcohol, diagnóstico previo de laringitis crónica, diagnóstico de cáncer previo y RT previa, se presentaron en un porcentaje considerablemente menor $(16 \%, 10 \%, 9 \%$ y $6 \%$, respectivamente). En cuanto a la histología, el 93\% de los tumores fue epidermoide, $5 \%$ verrucoso y $2 \%$ adenocarcinoma.

El $53 \%$ de los pacientes $(n=51)$ fue sometido a LP, mientras que el $47 \%(n=45)$ recibió RT. El Gráfico 1 muestra la modalidad de tratamiento según el estadio tumoral, predominando levemente la radioterapia en los carcinomas in situ y

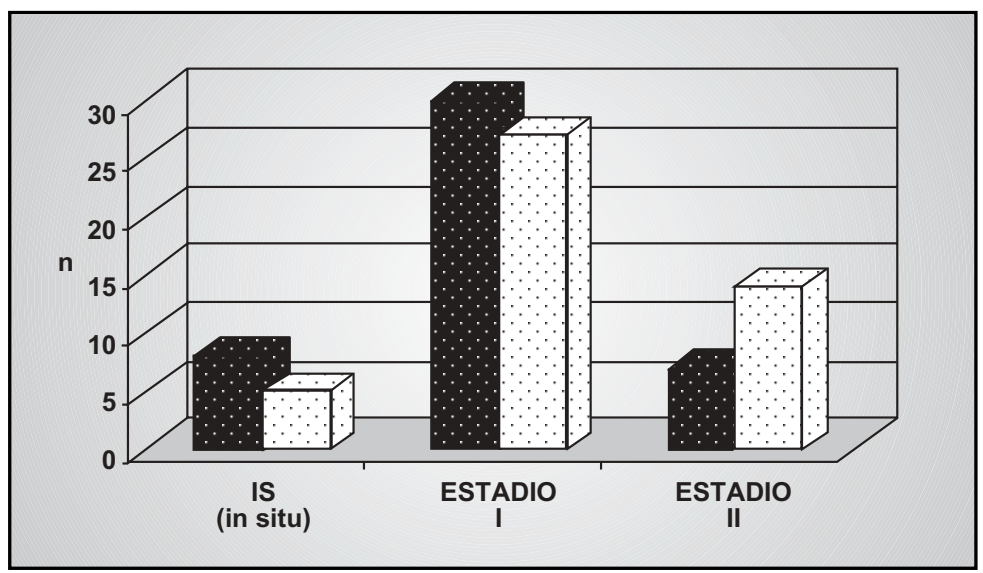

Gráfico 1. Modalidad de tratamiento según estadio de cáncer de laringe. 
etapa I, y la cirugía parcial de laringe en estadio II. En cuanto a la técnica quirúrgica, la laringectomía fronto-lateral fue la más realizada (Gráfico 2).

La complicación posoperatoria precoz más frecuente fue la formación de granulomas en el ostoma traqueal, en el $16 \%$ de los pacientes; mientras la complicación tardía más frecuente fue la falta de cierre espontáneo de la traqueostomía, en $16 \%$ (Tabla 1).

Se constató recidiva del cáncer en uno de los pacientes intervenidos quirúrgicamente $(2 \%)$ y en 4 de los tratados con RT (9\%). De estos últimos, dos pudieron ser sometidos a laringectomía parcial, mientras en los otros dos casos se debió realizar laringectomía total. La sobrevida actual es de $91 \%$ para los pacientes tratados con RT y $100 \%$ para laringectomía parcial.

De los 51 pacientes con cáncer glótico en quienes se les hizo una laringectomía frontolateral, el $75 \%$ (38 casos) de ellos tenían compromiso de la comisura anterior; en cambio, de los 45 pacientes que recibieron radioterapia, el $25 \% \quad(n=11)$ tenía compromiso de la comisura anterior (Tabla 2). Se consideró compromiso de comisura anterior cuando a la laringoscopía directa se observó que el

Tabla 1. Complicaciones de tratamiento quirúrgico en pacientes con cáncer laríngeo precoz

\begin{tabular}{|ll|}
\hline Complicaciones cirugía $(\mathrm{n}=51)$ & \\
\hline Precoces & $\mathrm{n}$ \\
\hline Infección traqueostoma & 4 \\
Granulomas & 8 \\
Tardías & 5 \\
Trastorno deglución & 8 \\
Traqueostoma persistente & 1 \\
Estenosis laríngea & \\
\hline
\end{tabular}

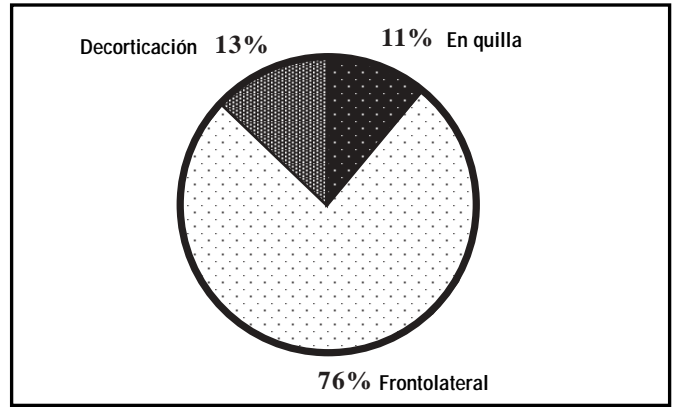

Gráfico 2. Tipo de técnica quirúrgica utilizada en pacientes tratados con cirugía parcial de laringe.

tumor comprometía la CA, descartando aquellos casos en los cuales sólo existía edema. Desde el año 1990 todos los pacientes con cáncer precoz de cuerda con compromiso de la CA tenían tomografía computada de cuello y en ninguno de ellos se observó destrucción del cartílago a nivel de CA. Los que tenían compromiso de cartílago por imágenes, ya habían sido catalogados clínicamente como cáncer avanzado.

En cuanto al resultado del tratamiento, se observa que todos los pacientes que presentaron recidiva de cáncer laríngeo tenían previamente compromiso de la CA. En el análisis según modalidad de tratamiento utilizado, existe mayor recidiva con compromiso de la comisura anterior cuando se utiliza RT ( $p=0.01$ ), no existiendo diferencias significativas en recidiva en los pacientes sometidos a cirugía parcial (Tabla 3).

\section{DISCUSIÓN}

En el abordaje terapéutico del cáncer laríngeo precoz tanto la cirugía parcial de laringe como la radioterapia son alternativas unimodales de tratamiento adecuadas. Si bien en algunos estudios existen diferencias en cuanto al control local de la

Tabla 2. Compromiso de comisura anterior en pacientes tratados por cáncer glótico precoz

\begin{tabular}{|lcc|}
\hline Modalidad tratamiento & Compromiso comisura anterior & Comisura anterior normal \\
\hline Cirugía parcial $(n=54)$ & $76 \%(n=41)$ & $24 \%(n=13)$ \\
Radioterapia $(n=45)$ & $24 \%(n=11)$ & $76 \%(n=34)$ \\
\hline
\end{tabular}


Tabla 3. Recidiva de cáncer laríngeo de acuerdo a tratamiento realizado y compromiso de comisura anterior

\begin{tabular}{|llc|}
\hline Modalidad tratamiento & Comisura anterior & Recidiva \\
\hline Cirugía parcial $(n=54)$ & Comprometida $(n=41)$ & $5 \%$ \\
& Normal $(n=13)$ & $0 \%$ \\
Radioterapia $(n=45)$ & Comprometida $(n=11)$ & $18 \%$ \\
& Normal $(n=34)$ & $0 \%$ \\
\hline
\end{tabular}

enfermedad, siendo mejor la radioterapia en unas ${ }^{19}$ o la cirugía en otras ${ }^{20,28}$, ambas modalidades son igualmente efectivas en términos de conservación laríngea y tasa de sobrevida ${ }^{14,15,17,29}$.

Las principales series nacionales publicadas de cáncer larínge $0^{3,30-32}$ incluyen en su mayoría estadios avanzados, propios de la realidad nacional, por lo que es imposible comparar los resultados con esta serie. En nuestra casuística, la relación hombre:mujer es más estrecha (4:1) de lo que muestran otras publicaciones nacionales ${ }^{3,30-32}$, Io que se aproxima más a la realidad internacional ${ }^{33}$.

En esta serie de pacientes tratados a lo largo de 25 años, las frecuencias estimadas de recidiva son menores al $10 \%$ tanto para RT como LP, mientras la sobrevida es superior al $90 \%$ en ambas alternativas de tratamiento. En general, pueden considerarse comparables con la literatura extranjera, punto que resulta destacable si se considera que la serie abarca pacientes tratados en la década de los ' 80 , tiempo desde el cual se ha optimizado la RT como herramienta terapéutica y la experiencia quirúrgica ha sido mayor.

Una publicación nacional aborda el cáncer laríngeo de ubicación glótica en etapa precoz mediante cirugía transoral con empleo de láser logrando buenos resultados ${ }^{34}$. Esta herramienta terapéutica corresponde indudablemente a un avance, validado por múltiples experiencias internacionales, pero no se encuentra disponible masivamente en nuestro país y su incorporación implica una curva de aprendizaje inicial y el conocer los resultados a mediano y largo plazo en series locales. Lo anterior, junto al hecho de ser una técnica cara, respaldaría el planteamiento respecto a que quien realice esta técnica debería tener una certificación de competencia previa.
En relación a las complicaciones quirúrgicas, se ha reportado la aparición de infección, hemorragia, fístula faríngea, aspiración y neumonía ${ }^{35}$. En este estudio se presentaron complicaciones en un bajo porcentaje de los pacientes sometidos a laringectomía parcial, siendo las más frecuentes la traqueostomía persistente y la aparición de granulomas, ambas de resolución sencilla y que no complican habitualmente la evolución del paciente.

Respecto al compromiso de la CA, según nuestra experiencia constituye un factor de riesgo para recidiva de cáncer laríngeo, presentando diferencias estadísticamente significativas en el grupo sometido a RT, no así en cirugía parcial. Debe recordarse que en este punto las modalidades de tratamiento no son comparables, debido a la selección de pacientes efectuada previamente de acuerdo al protocolo seguido por el autor.

Puesto que las modalidades de tratamiento del cáncer laríngeo precoz son similares en cuanto a recurrencia y sobrevida, es necesario considerar otros factores para la elección del tratamiento, en especial la calidad de vida, factibilidad de los controles, personalidad del paciente en cuanto a si es capaz de dejar de fumar o no y el costo. En general se considera que la RT implicaría un mayor costo que la cirugía ${ }^{29}$; sin embargo, en cuanto a calidad de vida, la mayoría de los estudios reportan que mediante la RT se logra una mejor preservación de la voz ${ }^{35,36}$. Considerar múltiples aspectos, tales como extensión de la enfermedad, condición general del paciente, uso vocal, experiencia del equipo quirúrgico, disponibilidad de RT adecuada, etc., es necesario para escoger el esquema de tratamiento adecuado, el que sigue correspondiendo en la actualidad a un acuerdo informado entre el paciente y el médico tratante. 


\section{BIBLIOGRAFÍA}

1. FerLito $A$. The natural history of early vocal cord cancer. Acta Otolaryngol 1995; 115(2): 345-7.

2. Koufman JA, Burke $A$. The etiology and pathogenesis of laryngeal carcinoma. Aolaryngologic Cin North Am 1997; 30 (1): 1-19.

3. Celedón C, López R, Büchi M. Cáncer de laringe e hipofaringe. Rev otorrinolaringol 1981; 41: 4550.

4. Falk RT, Pickle LW, Brown LM, Mason TJ, Buffler PA, FraumenI JF JR. Effect of smoking and alcohol consumption on laryngeal cancer risk in coastal Texas. Cancer Res 1989; 49 (14): 4024-9.

5. Guenel P. A study of the interaction of alcohol drinking and tobacco smoking among French cases of laryngeal cancer. $J$ Epidemiol Community Health 1988; 42(4): 350-4.

6. PICCIRILLO JF. Importance of comorbidity in head and neck cancer. Laryngoscope 2000; 110 (4): 593-602.

7. Mork J, Lie AK, Glattre e et al. Human papillomavirus infection as a risk factor for squamous-cell carcinoma of the head and neck. NEngl J Med 2001; 344 (15): 1125-31.

8. Celedón C. Uso del interferon en la papilomatosis laríngea. Rev Atorrinolaringol Or Cabeza Quello 1995; 55: 45-9.

9. El-Serag hB, Hepworth eJ, Lee P, et al. Gastroesophageal reflux disease is a risk factor for laryngeal and pharyngeal cancer. Am J Gastroenterol 2001; 96 (7): 2013-8.

10. Galli J, Cammarota G, Volante M, De Corso E, Almadori G, Paludetti G. Laryngeal carcinoma and laryngo-pharyngeal reflux disease. Acta Aorhinolaryngol Ital 2006; 26 (5): 260-3.

11. Celedón C, Naquera N, Reveco J. Azul de toluidina y cáncer laríngeo. Rev Otorrinolaringol Or Ca beza Quello 1984; 44: 8-11.

12. Celedón C, Villarroel L, Bozan H. Tratamiento del cáncer precoz de cuerda vocal. Rev Otorrinolaringol Cir Cabeza Quello 1988; 48: 83-7.

13. Johnson JT. Carcinoma of the larynx: selective approach to the management of cervical lymphatics. Ear Nose Throat $J$ 1994; 73 (5): 303-5.
14. Jones AS, Fish B, Fenton JE, Husband DJ. The treatment of early laryngeal cancers (T1-T2 N0): Surgery or irradiation? Head Neck 2004; 26 (2): 127-35.

15. Nguyen C, Naghibzadeh B, Black MJ, Rochon L, Shenouda G. Carcinoma in situ of the glottic larynx: Excision or irradiation? Head Neck 1996; 18 (3): 225-8.

16. Spriano G, Antognoni P, Piantanida $R$ et al. Conservative management of T1-T2NO supraglottic cancer: A retrospective study. Am J Otolaryngol 1997; 18 (5): 299-305.

17. BACK G, Sood S. The management of early laryngeal cancer: options for patients and therapists. Ourr Opin Otolaryngol Head Neck Surg 2005; 13(2): 85-91.

18. Cragle SP, Brandenburg J. Laser cordectomy or radiotherapy: cure rates, communication, and cost. Otolaryngol Head Neck Surg 1993; 108(6): 648-54.

19. Spector JG, Sessions DG, Chao KS et al. Stage I (T1 NO M0) squamous cell carcinoma of the laryngeal glottis: therapeutic results and voice preservation. Head Neck 1999; 21(8): 707-17.

20. Magnano M, Cavalot Al, Gervasio CF et al. Surgery or radiotherapy for early stages carcinomas of the glottic larynx. Tumori 1999; 85(3): 188-93.

21. Ferlito A, Bradley PJ, Rinaldo $A$. What is the treatment of choice for $\mathrm{T} 1$ squamous cell carcinoma of the larynx? J Laryngol Col 2004; 118(10): 747-9.

22. Chen MF, Chang JT, tsang nM et al. Radiotherapy of early-stage glottic cancer: analysis of factors affecting prognosis. Ann Ool Rhinol Laryngol 2003; 112(10): 904-11.

23. Zouhair A, Azria D, Coucke P et al. Decrease local control following radiation therapy alone in early-stage glottic carcinoma with anterior comissure extension. Strahlenther Onkol 2004; 180 (2): 84-90.

24. Stoeckli SJ, Schnieper I, Huquenin P et al. Early glottic carcinoma treatment according to patients preferences. Head Neck 2003; 25(12): 1051-6.

25. Pradhan SA, Pal PS, Neeli SI, D'Cruz AK. Transoral laser surgery for early glottic cancers. Arch Aolaryngol Head Neck Surg 2003; 129(6): 623-5. 
26. Zeitels SM, Hillman RE, Franco Ra, Buntino GW. Voice and treatment outcome from phonosurggrical management of early glottic cancer. Ann Otol Rhinol Laryngol (suppl) 2002; 190: 3-20.

27. Steiner W, Ambrosch P, Rödel RM, Kron M. Impact of anterior comissure involvement on local control of early glottic carcinoma treated by laser microresection. Laryngoscope 2004; 114 (8): 1485-91.

28. Markou K, Nikolaou A, Nalbadian M, Petridis D, Nicolaidis V, DaniliLidis I. How often is total laryngectomy necessary for the treatment of $\mathrm{T} 1$ failures after radiotherapy or cordectomy? Eur Arch Aorhinolaryngol 2002; 259(1): 4-10.

29. Mlynarek A, Kost K, Gesser R. Radiotherapy versus surgery for early T1-T2 glottic carcinoma. J Otolaryngol 2006; 35(6): 413-9.

30. Contreras JM, Niklitschek E, Paredes A, Maass JC. Cáncer laríngeo-hipofaríngeo. Evaluación en el Hospital San Juan de Dios. Rev Otorrinolaringol Or Cab Quello 2001; 61: 19-30.

31. Contreras JM; Liebner A, Calabresse R, Plandiura J. Cáncer de laringe e hipofaringe: evaluación de los años 1980 a 1988. Rev Otorrinolaringol Or Cab Cuello 1989; 50: 25-33.

32. Pradenas M, Vallejos MP, Rahal M, Cisternas A. Cáncer de laringe e hipofaringe. Experiencia del Hospital Barros Luco-Trudeau 1990-2001. Rev Atorrinolaringol Cir Cabeza Cuello 2004; 64: 13-20.

33. DeRienzo DP, Greenberg SD, Fraire Ae. Carcinoma of the larynx: Changing incidence in women. Arch Otolaryngol Head Neck Surg 1991; 117 (6): 681-4.

34. Ortega A, Olcese L, Zanolli L, Lillo S. Manejo endoscópico de la patología glótica maligna inicial. Rev Aorrinolaringol Cir Cabeza Quello 2005; 65: 197-202

35. Bron LP, Soldati D, Zouhair A et al. Treatment of early stage squamous-cell carcinoma of the glottic larynx: Endoscopic surgery or cricohyoidoepiglottopexy versus radiotherapy. Head Neck 2001; 23(10): 823-9.

36. Foote RL, Buskirk SJ, Grado GL, Bonner JA. Has radiotherapy become too expensive to be considered a treatment option for early glottic cancer? Head Neck 1997; 19(8): 692-700. 